\title{
Correlations between Muscle Mass, Muscle Strength, Physical Performance, and Muscle Fatigue Resistance in Community-Dwelling Elderly Subjects
}

\author{
Elizabeth, Vitriana, Irma Ruslina Defi \\ Department of Physical Medicine and Rehabilitation, Faculty of Medicine, Universitas Padjadjaran-Dr. Hasan \\ Sadikin General Hospital
}

\begin{abstract}
Objective: To determine the correlations between muscle mass, muscle strength, physical performance, and muscle fatigue resistance in communitydwelling elderly people in order to elucidate factors which contribute to elderly's performance of daily activities.

Methods: A cross-sectional study was conducted on community-dwelling elderly in Bandung from September to December 2014. One hundred and thirty elderly, 60 years old or above, were evaluated using bioelectrical impedance analysis to measure muscle mass; grip strength to measure muscle strength and muscle fatigue resistance; habitual gait speed to measure physical performance; and Global Physical Activity Questionnaire (GPAQ) to assess physical activity.

Results: There were significant positive correlations between muscle mass $(r=0,27, p=0,0019)$, muscle strength $(r=0,26, p=0,0024)$, and physical performance $(\mathrm{r}=0,32, \mathrm{p}=0,0002)$ with muscle fatigue resistance. Physical performance has the highest correlation based on multiple regression test $(p=0,0025)$. In association with muscle mass, the physical activity showed a significant positive correlation $(r=0,42, p=0,0000)$. Sarcopenia was identified in $19(14.61 \%)$ of 130 subjects.
\end{abstract}

Received:
August 25, $2015 \quad$ Conclusions: It is suggested that muscle mass, muscle strength, and physical performance influence muscle fatigue resistance.

Revised:

December 31, 2015 Keywords: Community-dwelling, elderly, muscle fatigue resistance, muscle Accepted:

February 26, 2016 mass, sarcopenia

IJIHS. 2016;4(1):32-7

\section{Introduction}

One of the most serious consequences of ageing is its effects on skeletal muscle; particularly in the form of progressive loss of muscle mass and function which impacts the quality of life, and, ultimately, survival. ${ }^{1}$ In 1989, Irwin Rosenberg proposed the term 'sarcopenia' (Greek 'sarx' or flesh and 'penia' or loss) to describe agerelated decrease of muscle mass. ${ }^{2}$ European Working Group on Sarcopenia in Older People (EWGSOP) defines sarcopenia as a syndrome characterized by progressive and generalized

\footnotetext{
Correspondence:

Elizabeth, Department of Physical Medicine and Rehabilitation, Faculty of Medicine, Universitas Padjdajaran-Dr. Hasan Sadikin General Hospital Jl. Pasteur No. 38, Bandung, Indonesia e-mail: setiawanelizabeth@gmail.com
}

loss of skeletal muscle mass and strength with a risk of adverse outcomes, such as physical disability, poor quality of life, and death. The operational definition recommends using the presence of both low muscle mass and low muscle function (strength or performance) for the diagnosis of sarcopenia. ${ }^{3}$

Some of the mechanisms and determinants involved in muscle wasting during ageing involve hormonal changes. ${ }^{1}$ Several studies have indicated age-related endocrine defects such as decreases in anabolic hormones such as testosterone, estrogen, growth hormone (GH), and insulin-like growth factor-I (IGF-I). ${ }^{4}$ Contributors to the aging-related loss of muscle mass are also explained by oxidative stress that may lead to an alteration in the balance between mitochondrial fission and fusion as well as the activation of the apoptotic pathway. 
Skeletal muscles will undergo an age-related atrophy due to the decrease of the nuclear domain. This explains the more remarkable reduction in the size of type II muscle fibers than that of the type I muscle fibers. ${ }^{5}$

Aging reduces the force-generating capacity or the strength of the skeletal muscles. As a result, many older people experience some difficulties in performing their daily activities. Recent research indicates that the observed loss of force production in older people is primarily due to the result of muscle atrophy and alterations in the percentage of contractile tissue within muscle. ${ }^{6}$

Muscle size, strength, body composition, and maximum aerobic capacity (VO2 peak) are the components of walking that may be associated with mobility in elderly people. These components change with age and are likely to be predictors of slow gait speed. Specifically, maximum aerobic capacity has been demonstrated to be a strong predictor of mobility. ${ }^{7}$

Fatigue resistance, or the ability to produce sustained skeletal muscle force output, may be crucial for high-level sports performance, and becomes essential for proper daily functioning later in life. With advancing age, both muscle volume and muscle strength decrease. Consequently, sustained daily activities at older ages need muscle activities closer to one's individual maximum. ${ }^{8}$

Weakness may occur due to the sustained atrophy of muscles experienced during the ageing process. It is interesting, however, that the observed relative maximum force or power of atrophied muscle shows a greater resistance to fatigue. The study of whether and how older skeletal muscle resists fatigue has become an important and active area of research. ${ }^{9}$

The correlations between muscle mass, muscle strength, and physical performance with muscle fatigue resistance are interesting topics for researchers. Hence, this study has the objective to study those correlations, especially among community-dwelling elderly subjects in Bandung.

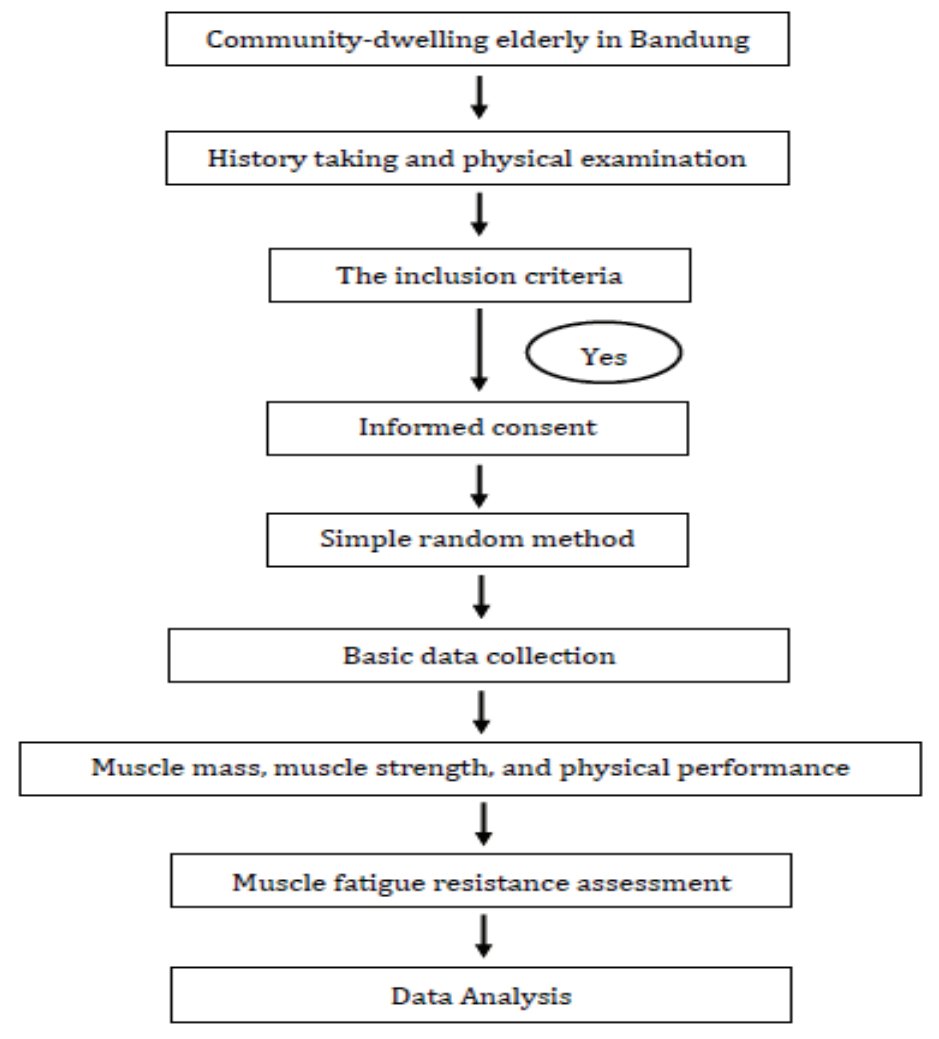

Fig. 1 Study Pattern 


\section{Methods}

This study was a cross-sectional study with simple random sampling that analyzed 130 60-year-old or above community-dwelling subjects in Bandung during the period of September-December 2014. Subjects were included if they were able to walk inside and outside of the house independently without assistive device, cooperative and were willing to participate the study, sign the informed consent form, and did not live in nursing home. Subjects were excluded when they have dementia (Mental State Examination $<27 / 30$ ); disorders of cardiorespiratory and cardiovascular systems (hypertension stage II according to JNC 7 criterion); musculoskeletal or neuromuscular disorders that cause inability to walk independently based on history and physical examination; artificial metal implants which would interfere with the accuracy of the results of the Body Impedance Analyzer (BIA) such as pacemakers; hormone therapy; long term steroid therapy; diseases that can cause loss of muscle mass such as malignancy, chronic infection, congestive cardiomyopathy, and renal impairment; and lesion on the lower limb which causes pain or movement disorders.

Each subjects underwent height and body weight measurement as well as GPAQ for physical activity assessment. The muscle mass was evaluated by bio impedance analysis using a segmental multiple frequency analyser (BC-601 Bio Impedance Analyzer Tanita ${ }^{\circledR}$ ) to measure upper and lower limb muscle mass (kg). Appendicular muscle mass (AMM) was derived as the sum of the muscle mass of the arms and the legs. The result of AMM was converted to appendicular muscle mass index (AMI) by dividing with height in meters squared $(\mathrm{kg} / \mathrm{m} 2){ }^{10}$

Muscle strength was assessed by hand grip strength, which was measured using a Jamar ${ }^{\circledR}$ hydraulic hand dynamometer in which subject was standing with the shoulder adducted and neutrally rotated, the elbow flexed at 900 , the forearm in neutral position and the wrist in light extension ( 0 to 300 ). The subject was then instructed to squeeze the rubber bulb of Jamar ${ }^{\circledR}$ hydraulic hand dynamometer as hard as possible. The score was recorded for dominant hand, as it corresponds to the real potential maximum strength of a subject. ${ }^{8}$

To assess muscle fatigue resistance, the subject was asked to squeeze the rubber bulb of Jamar hydraulic hand dynamometer as hard as possible and instructed to maintain this pressure as long as possible. The observer verified that the starting strength corresponded to the maximum grip strength tested before. The time (in seconds) until the pressure diminished to $50 \%$ of the maximum grip strength was recorded for dominant hand. The same position of the subject's arm was adopted as for the grip strength test. Subject was instructed to maintain the elbow in $90^{\circ}$ flexion and was not allowed to see the readings on the dynamometer at any time during the procedure. ${ }^{8}$

The physical performance was assessed by habitual gait speed. Subject was asked to

Table 1 Subject Characteristics

\begin{tabular}{|c|c|c|c|c|c|c|}
\hline & n (\%) & $\begin{array}{c}\text { Age } \\
\text { (years) }\end{array}$ & $\begin{array}{c}\text { Body Mass } \\
\text { Index } \\
(\mathrm{kg} / \mathrm{m} 2)\end{array}$ & $\begin{array}{c}\text { Appendicular } \\
\text { Muscle Mass } \\
\text { Index } \\
\text { (kg/m2) }\end{array}$ & $\begin{array}{l}\text { Muscle } \\
\text { Strength } \\
\text { (kg) }\end{array}$ & $\begin{array}{c}\text { Habitual } \\
\text { Gait Speed } \\
\text { (m/s) }\end{array}$ \\
\hline Total & $130(100)$ & $68.7 \pm 6.6$ & $25.2 \pm 3.8$ & $7.2 \pm 1.2$ & $16.0 \pm 5.1$ & $0.8 \pm 0.2$ \\
\hline \multicolumn{7}{|l|}{ Male } \\
\hline Total & $22(16.9)$ & $71.1 \pm 7.4$ & $25.6 \pm 3.6$ & $9.2 \pm 1.2$ & $19.7 \pm 6.4$ & $0.9 \pm 0.2$ \\
\hline Sarcopenia & $3(2.3)$ & $69.7 \pm 7.6$ & $22.6 \pm 0.5$ & $8.1 \pm 0.4$ & $19.3 \pm 3.0$ & $0.8 \pm 0.1$ \\
\hline Normal & $19(14.6)$ & $71.4 \pm 7.6$ & $26.1 \pm 3.7$ & $9.3 \pm 1.2$ & $19.7 \pm 6.8$ & $0.9 \pm 0.2$ \\
\hline \multicolumn{7}{|l|}{ Female } \\
\hline Total & 108(83.1) & $67.6 \pm 6.3$ & $25.2 \pm 3.8$ & $6.8 \pm 0.7$ & $15.3 \pm 4.4$ & $0.8 \pm 0.2$ \\
\hline Sarcopenia & $16(12.3)$ & $69.2 \pm 7.4$ & $21.2 \pm 2.6$ & $6.1 \pm 0.2$ & $15.4 \pm 4.4$ & $0.8 \pm 0.1$ \\
\hline Normal & $92(70.8)$ & $67.3 \pm 6.1$ & $25.9 \pm 3.6$ & $7.0 \pm 0.6$ & $15.3 \pm 4.5$ & $0.8 \pm 0.2$ \\
\hline
\end{tabular}


Table 2 Correlations of Muscle Mass, Muscle Strength, and Physical Performance with Muscle Fatigue Resistance

\begin{tabular}{lll}
\hline \multicolumn{1}{c}{ Correlation } & rs* & p Value \\
\hline Muscle fatigue resistance $><$ Appendicular muscle mass index (AMI) & 0,27 & $0,0019^{* *}$ \\
Muscle fatigue resistance $><$ Muscle strength & 0,26 & $0,0024^{* *}$ \\
Muscle fatigue resistance $><$ Physical performance & 0,32 & $0,0002^{* *}$ \\
\hline
\end{tabular}

Note: ${ }^{\top}$ J spearman correlation test

**) Significant, $p \leq 0,05$

walk straight ahead for $15 \mathrm{~m}$ at his/her usual speed. Habitual gait speed was derived over 6 m course. ${ }^{2}$

This study uses descriptive analysis for subject characteristics as well as the Spearman correlation analysis and the multiple linear regression for the analysis on the correlation between muscle mass, muscle strength, and physical performance with the muscle fatigue resistance in elderly. The significance statistical test results was determined based on a $p$ value $\leq 0.05$.

The study has been authorized in ethical committee of Hasan Sadikin General Hospital to be conducted in allocated subjects.

\section{Results}

Subjects' characteristics demonstrated 130 subjects of age 60-85 years old, mostly females, and have overweight body mass index which were described (Table 1). In this study, 2.3\% of men and $12.3 \%$ of women were classified as having sarcopenia according to the cutoff values recommended by Asian Working Group for Sarcopenia (AWGSOP). Overall, the subjects of this study used their right hand as the dominant hand.

Correlations between muscle mass, muscle strength, and physical performance with muscle fatigue resistance were analyzed using Spearman Corelation test and the results were shown as significant with a $p$ value of $\leq 0.05$ (Table 2).

Results of the multiple regression test for assessing the correlations of muscle mass, muscle strength, and physical performance with muscle fatigue resistance are shown in table 3. Muscle strength could not be entered to the test because it has a $p$ value that was less significant compared with AMI and physical performance. The last two factors were able to represent the muscle strength. Physical performance was shown to have the highest correlation with muscle fatigue resistance.

Correlation between muscle mass and physical activity was also analyzed using the Spearman Corelation test and was shown as significant with a p value of $\leq 0.05$ (Table 4 ).

\section{Discussion}

This study involved 130 community-dwelling elderly subjects in Bandung who met the inclusion criteria. The study was conducted from September to December 2014. The AWGSOP guideline was used to define sarcopenia in this study.

The mean age of the subjects was 68.7 years with the youngest subject was 60 years old and the oldest was 85 years old. More female subjects participated in the study with 108 females $(83.1 \%)$ compared to 22 males $(16.9 \%)$. This gender distribution is consistent with the Indonesia Population Projection 2010-2035, which most elderly above 60 years old are females and that women's life expectancy are higher than men. ${ }^{11}$

The body mass index of the subjects with sarcopenia was lower compared to the normal subjects. These may result in low nutrient intake, which is an important risk factor in the development of sarcopenia. In particular, protein intake has a major influence on skeletal muscle metabolism. Inadequate protein intake is one of the major mechanisms underlying sarcopenia. ${ }^{12}$

Muscle mass was measured by BIA and the range of appendicular muscle mass index of both community-dwelling male and female elderly in Bandung was above the cutt-off values recommended by AWGSOP. The AWGSOP recommends cut-off values of sarcopenia for muscle mass measurement by BIA of AMI $<8.87 \mathrm{~kg} / \mathrm{m} 2$ for males and AMI $<6.42 \mathrm{~kg} / \mathrm{m} 2$ for females.

The grip strength was used to measure muscle strength. The sarcopenia cut-off 
values for handgrip strength recommended by AWGSOP are $<22.4 \mathrm{~kg}$ for males and $<14.3 \mathrm{~kg}$ for female.s The range of grip strength of male in this study was below the cut-off value, while the grip strength of female was above the cutoff value.

The algorithm that is based on gait speed measurement suggested by the EWGSOP was used in this study for screening sarcopenia and physical performance evaluation. A cutoff point of $>0.8 \mathrm{~m} / \mathrm{s}$ identifies the risk for sarcopenia. Most of the subjects in this study have a habitual gait speed on the border of cutoff values. ${ }^{2}$

A previous study in Asia reported that the prevalence of sarcopenia is higher in female subjects $(19.9 \%$ in female and $17.2 \%$ in male), which was relevant to the finding of this study of about $12.3 \%$ in female and $2.3 \%$ in male. ${ }^{4}$ Setiati, et al. also reported $8 \%$ low grip strength and $2.8 \%$ limited mobility in 251 geriatric outpatients in Cipto Mangunkusumo Hospital, Jakarta, Indonesia. ${ }^{15}$

Spearman correlation test analysis results with a degree of confidence of $95 \%$ indicates that there are positive correlations between muscle mass, muscle strength, and physical performance with muscle fatigue resistance statistically. They might be due to more motor units that must be recruited and at higher frequency of excitation for atrophied muscles to perform a given motor task. ${ }^{16}$ As aerobic capacity declines with age, the exertion associated with habitual gait speed increases. A slowing of walking speed may be a response to increased perception of exertion. ${ }^{7}$

This study also showed that physical activity correlates with muscle mass. A study has also reported a disruption in the balance between protein synthesis and breakdown in which muscle protein anabolism is reduced and catabolism is increased. ${ }^{17}$

This study did not classify and correlate the parameters between gender and age range. There is also no cut-off point for the assessment of muscle mass, muscle strength, and physical performance in Indonesia, in general, and in Bandung, in particular. In addition, the biomolecular mechanisms of sarcopenia and muscle fatigue resistance relationship in details are still unknown and more in-depth studies, both in vivo and in vitro, are needed.

In summary, this study shows that sarcopenia, which is defined by muscle mass, muscle strength, and physical performance, had a significant correlation with muscle fatigue resistance in community-dwelling elderly subjects in Bandung, Indonesia. Evidently, physical performance has the highest correlation with muscle fatigue resistance compared with others. The study also revealed the significantly correlation between muscle mass with physical activity.

\section{References}

1. Lynch G S. Sarcopenia - Age-Related Muscle Wasting and Weakness Mechanisms and Treatments. Dordrecht Heidelberg London New York: Springer; 2011.

2. Cruz-Jentoft A J, Baeyens J P, Bauer J M, Boirie Y, Cederholm T, Landi F, et al. Sarcopenia: European consensus on definition and diagnosis. Age and Ageing. 2010;39: 412-423

3. Cruz-Jentoft A J, Morley J E. Sarcopenia. United Kingdom: Wiley-Blackwell; 2012.

4. Sakuma K, Yamaguchi A. Sarcopenia and agerelated endocrine function. Int J Endocrinol. 2012; [cited 2015 Jan 10]:[about 10 p.]. Available from: http://www.ncbi.nlm.nih.gov/ pmc/articles/PMC3368374/.

5. Kwan P. Sarcopenia, a neurogenic syndrome? Journal of Aging Research. 2013; [cited 2015 Jan 10]:[about 10 p.]. Available from: http://www.ncbi.nlm.nih.gov/pmc/articles/
PMC3610356/.

6. Williams GN, Higgins MJ, Lewek MD. Aging Skeletal muscle: physiologic changes and the effects of training. Phys Ther. 2002;82(1):62-8.

7. Fiser WM, Hays NP, Rogers SC, Kajkenova O, Williams AE, Evans CM, et al. Energetics of walking in elderly people: factors related to gait speed. J Gerontol A Biol Sci Med Sci. 2010;65(12):1332-7.

8. Bautmans I, Mets T. A fatigue resistance test for elderly persons based on grip strength: reliability and comparison with healthy young subjects. Aging Clin Exp Res. 2004;17(3):21722.

9. Williams C, Ratel S. Human Muscle Fatigue. New York: Routledge; 2010.

10. Tanimoto $Y$, Watanabe M, Sun W, Sugiura Y, Tsuda Y, Kimura M, et al. Association between sarcopenia and higher-level functional capacity 
in daily living in community-dwelling elderly subjects in Japan. Arch Gerontol Geriatr. 2012;55(2):9-13.

11. Badan Perencanaan Pembangunan Nasional Badan Pusat Statistik United Nation Population Fund. Proyeksi Penduduk Indonesia 20102035. Jakarta. 2013.

12. Rom O, Kaisari S, Aizenbud D, Reznick AZ. Lifestyle and sarcopenia-etiology, prevention, and treatment. Rambam Maimonides Med J. 2012;3(4):1-12.

13. Chen LK, Liu LK, Woo J, Assantachai P, Auyeung TW, Bahyah KS, et al. Sarcopenia in Asia: consensus report of the Asian Working Group for Sarcopenia. 2014;15(2):95-101.

14. Tanimoto Y, Watanabe M, Sun W, Hirota C,
Sugiura Y, Kono R, et al. Association between muscle mass and disability in performing instrumental activities of daily living (IADL) in community-dwelling elderly in Japan. Arch Gerontol Geriatr. 2011;54(2)230-3.

15. Setiati S. Geriatric Medicine, Sarkopenia, Frailty dan Kualitas Hidup Pasien Usia Lanjut: Tantangan Masa Depan Pendidikan, Penelitian dan Pelayanan Kedokteran di Indonesia. eJKI. 2013;1(3):234-42.

16. Farrel P A, Joyner M J, Caiozzo V J. ACSM's Advanced Exercise Physiology. Baltimore: Lippincott Williams \& Wilkins; 2012.

17. Narici M V, de Boer. Disuse of the musculoskeletal system in space and on earth. Eur J Appl Physiol. 2011;111(3):403-20. 